violets, pour l'examen systématique de tous les échantillons reçus dans les lactariums. Ce double contrôle aurait comme avantage d'accroître la sécurité en une matière sur l'importance de laquelle il est inutile d'insister.

(Ce travail a été effectué avec l'aide de l'Institut National d'Hygiène, Professeur Bugnard.)

(Clinique de Puériculture de la Faculté de Paris, Professeur Marcel Lelong.)

\title{
POUR LA PASTEURISATION OBLIGATOIRE DU LAIT DE CONSOMMATION
}

\author{
par \\ JEAN PIEN \\ Docteur es Seiences \\ (fin)
}

\section{$3^{\circ}$ Valeur alimentaire du lait pasteurisé}

a) Point de vue particulier de la digestibilité du lait chauffé.

Marfan s'exprime ainsi (loc. cit., p. 559 et suivantes) :

"En ce qui concerne la digestion stomacale, si le lait de vache " stérilisé par la chaleur ne subit pas les mêmes modifications que " le lait de femme, l'estomac le digère mieux que le lait de vache "cru.

"Si l'on juge la digestion intestinale d'après le degré d'assimila" tion des principes du lait, on voit qu'elle est au moins aussi bonne " avec le lait stérilisé qu'avec le lait cru.

"Si l'on étudie comparativement l'utilisation des principes " du lait de vache, on voit qu'elle est meilleure ou aussi bonne avec " le lait stérilisé ou bouilli qu'avec le lait cru. C'est ce que montrent "les recherches qui ont eu pour objet de mesurer la croissance " et d'évaluer les entrées et les sorties des divers principes du lait "chez des enfants ou de jeunes animaux alimentés soit avec du lait " cru, soit avec du lait pasteurisé, bouilli ou stérilisé. Les gains de "l'organisme en expérience sont les mêmes, quelle que soit la " manière dont le lait a été traité. Souvent même, quand il s'agit "des enfants, ils sont plus élevés avec les laits chauffés qu'avec le "lait eru."

(Travaux cités par Marfan : Bendix, Lange, W. Cronheim et E. Muller, Variot et Lorenz Monod, Sebileau, Raudnitz, Duclaux, Bodet, Schroeder.) 
b) Point de vue général de la valeur nutritive du lait pasteurisé. Etude de la croissance des animaux.

Schroeder (1913) dans une vaste expérience portant sur 600 cobayes nourris avec le lait de la mère, ou le lait de vache bouilli ou pasteurisé ou cru, aboutit aux conclusions suivantes : les cobayes nourris avec le lait de leur mère étaient supérieurs en poids et en pourcentage de survivants; ceux qui consommaient du lait de vache bouilli venaient après, mais étroitement suivis par les deux autres groupes (lait de vache cru ou pasteurisé) lesquels présentèrent des résultats identiques. Ces résultats confirmaient les recherches de Kelien sur les souris, de Lane Claypon sur les rats, de Rodet, Keller et Bruning sur les chiens, les pores et les chèvres.

L'Institut National de Recherches laitières de Reading (Angleterre) et l'Institut de Recherches Rowett, à Aberdeen, ont également étudié (1936) la valeur nutritive comparée du lait de vache cru et pasteurisé sur des rats. Il fut trouvé que la rétention du calcium et $\mathrm{du}$ phosphore, la digestibilité et la valeur biologique des protéines étaient identiques pour les deux types de lait. Egalement en utilisant comme critères les gains de poids, la longueur du corps et la composition du squelette des rats, aucune différence ne fut constatée entre le lait cru et le lait pasteurisé.

WILson et ses collaborateurs (1937) rapportent les résultats de leurs expériences d'alimentation de veaux pendant plus de deux ans à l'aide de lait cru et de lait pasteurisé. Les moyennes d'accroissement de poids du corps pour une durée de huit semaines furent (pour 100 animaux) : 61,2 livres pour les animaux alimentés au lait cru et 62,9 livres pour ceux alimentés au lait pasteurisé. Il n'était pas possible de distinguer de différence entre les deux groupes en ce qui concerne la condition, l'aspect extérieur ou d'autres points.

c) Point de vue général de la valeur nutritive du lait pasteurisé. Etude de la croissance des enfants.

Devant les discussions souvent sans base scientifique et presque toujours partiales qui opposaient sans cesse les partisans du lait cru et les défenseurs du lait pasteurisé, la Commission du Lait du Service de la Santé Publique des Etats-Unis a pris la position suivante :

"Pour réagir contre la propagande en faveur du lait cru, appuyée sur les affirmations sans base scientifique, la Commission mentionne plusieurs études selon lesquelles le lait pasteurisé a une valeur nutritive équivalente à celle du lait cru et insiste auprès du Service d'Hygiène Publique des Etats-Unis pour régler la question une fois pour toutes." 
En réponse à cet appel, le Service d'Hygiène du Bureau des Recherches laitières des Etats-Unis a immédiatement entrepris une vaste étude (dont furent chargés : Frank, Clark, Haskell, Miller, Moks et Thomas) dans le but de savoir notamment si les enfants qui consomment du lait cru se développent mieux que ceux qui boivent du lait pasteurisé.

L'expérience porta sur 3.637 enfants de dix mois à six ans (1.875 furent alimentés au lait pasteurisé et 1.762 au lait cru). Les Services de la Santé des Etats d'Alabama, Mississipi, Floride, Georgie, Caroline du Nord, Kentucky, Texas, Missouri, Orégon, Washington, collaborèrent avec les expérimenteurs du Service de la Santé Publique des Etats-Unis dans cette vaste enquête expérimentale poursuivie dans trente-neuf villes des Etats énumérés ci-dessus.

Les résultats de cette étude sensationnelle parurent dans "The Journal of the American Medical Association " du 29 octobre 1932, et peuvent être brièvement résumés comme suit :

L'étude ne révéla aucune différence sensible entre le poids moyen des enfants qui n'avaient consommé que du lait pasteurisé et le poids moyen de ceux qui avaient consommé du lait cru - et cela pendant une période de temps supérieure à la deuxième moitié de leur existence. La différence, insignifiante, était cependant en faveur des enfants qui n'avaient consommé que du lait pasteurisé.

Il en fut de même pour la comparaison des tailles : il n'y avait pas de différence sensible entre la taille moyenne des enfants soumis aux deux régimes expérimentés. La taille moyenne des enfants qui avaient consommé le lait cru était : $94 \mathrm{~cm}$. 9 ; la taille moyenne des enfants qui avaient consommé le lait pasteurisé était : $95 \mathrm{~cm} .2$. Si petite que fût la différence, elle était encore en faveur du lait pasteurisé.

En ce qui concerne la résistance aux infections on releva les proportions suivantes d'enfants atteints de maladies contagieuses ou de troubles intestinaux (pour 1.000 enfants) :

Nourris au lait pasteurisé Nourris au lait cru

$\begin{array}{lrr}\text { Scarlatine } \ldots \ldots \ldots \ldots \ldots \ldots \ldots & 23,0 & 41,4 \\ \text { Diphtérie } \ldots \ldots \ldots \ldots \ldots \ldots & 17,1 & 22,7 \\ \text { Troubles intestinaux } \ldots \ldots \ldots & 111,0 & 196,0\end{array}$

Ces résultats sont incontestablement en faveur du lait pasteurisé. La différence entre les deux types de laits peut s'expliquer soit par une diminution de la résistance aux infections des enfants consommant le lait cru, soit plus vraisemblablement par une contamination supplémentaire des enfants consommant ce lait. (Rappelons qu'il s'agit d'une expérience réalisée dans trente-neuf 
villes de dix Etats, ce qui donne à ces résultats une singulière valeur pratique.)

Une expérience analogue avait été poursuivie en Angleterre deux ans plus tôt (1930) sur des écoliers recevant du lait cru ou du lait pasteurisé en plus de leur régime habituel. L'expérience, dirigée par Leighton et McKinlay eut lieu dans le Lanarkshire et porta sur 5.000 enfants dans chaque groupe (avec 10.000 autres enfants ne recevant pas de lait et servant de témoins). Lés résultats furent notamment les suivants (concernant le poids et la taille) : l'apport de lait (cru ou pasteurisé) en complément de la ration habituelle donna une augmentation du gain de poids et de taille par rapport aux témoins ne recevant pas de lait; il n'y eut aucune évidence d'une supériorité quelconque du lait eru sur le lait pasteurisé (ni inversement) en ce qui concerne la croissance générale des enfants; la valeur de la pasteurisation se traduisit nettement dans l'élimination des infections.

Une autre expérience effectuée à Washington (D. C.) sur plusieurs centaines d'enfants en bas âge a fourni les résultats suivants : après dix-huit mois l'augmentation de poids par jour et par enfant était de 13 gr. 6096 pour ceux qui reçurent du lait oru et de 13 gr. 6768 pour ceux qui reçurent du lait pasteurisé.

LANE-CLAYPON observait déjà en 1916 que la majorité des auteurs concluaient qu'il n'y avait aucune preuve de l'infériorité du lait chauffé (bouilli ou pasteurisé) comparativement au lait cru et que, même, eertains auteurs concluaient que le lait cru était inférieur au lait bouilli. "Il n'y a aucune preuve, dit LANE-CLAYPON, " que le lait perde quoi que ce soit de sa valeur alimentaire par "l'ébullition et les travaux de nombreux expérimentateurs montrent "que les progrès obtenus avec le lait bouilli sont plus satisfaisants "en général qu'avec le lait cru. "

Enfin rappelons que l' "American Medical Milk Cómmission " a conclu depuis longtemps que "pour la nourriture des enfants, le lait ehauffé peut être considéré comme égal au lait cru. n

\section{$4^{\circ}$ Réponse à l'objection de l'ébullition domestique}

On fait quelquefois au principe de la pasteurisation l'objection suivante : "Pourquoi s'évertuer à pasteuriser puisque, en général, le consommateur fait bouillir le lait qu'il vient d'acheter?"

G. Thievlin a répondu récemment (1) à cette objection dans les termes suivants :

"Pour que, sur du lait quelconque, l'ébullition ait une action "valable il faut qu'elle se transforme en une véritable cuisson main-

(1) Conférence faite devant la Société de MLédecine et d'Hygiène le 17 avril 1950, à Casablanca. 
" tenue cinq minutes. Encore n'aboutirions-nous pas fatalement, en " agissant ainsi, à du bon lait.

"La " montée " du lait que l'on chauffe se produit aux environs « de 75 à $80^{\circ}$ et constitue une fausse ébullition n'offrant aucune "garantie.

"Si l'on ne brise pas obstinément la "peau " et la mousse qui " se forment, les microbes contenus dans cette mousse sont protégés " contre l'action de la chaleur."

"A un autre point de vue, le lait cru utilisé est de petit mélange " au taux de matière grasse inconstant et chaque jour inconnu. " Un contrôle généralisé de toutes les fournitures étant absolument "impraticable, la fraude, préjudiciable sur le plan alimentaire, "désastreuse pour l'hygiène, ne peut que rester impunie."

"Lorsque le lait dont dispose la ménagère est acidifié il ne " supporte pas l'ébullition. Pour éviter la coagulation intempestive, " il est alors simpliste de ne pas le faire bouillir ou, ce qui est égale" ment à craindre, de lui ajouter une pincée de bicarbonate de soude. "Méthodes aux conséquences tragiques: le défaut de chauffage " maintient tous les dangers présents, la neutralisation chimique "inverse le sens des phases bactériennes et favorise la putréfaction.

"Il est donc plus qu'aléatoire de compter hypocritement sur "l'ébullition domestique pour protéger la population contre les " méfaits du lait malpropre."

Nous avons nous-mêmes répondu à cette objection dans une publication ancienne (2). Nous disions notamment :

"Il faut bien noter que l'ébullition pratiquée par le consomma"teur n'a rien de commun avec la pasteurisation. La pasteurisation " a lieu dans les premières heures qui suivent la traite. La mise en "vente du lait cru, au contraire, ne peut se produire qu'après un " temps nettement plus long, nécessité par le transport, la distri" bution aux boutiques de vente, etc. L'ébullition au domicile du " consommateur a lieu encore plus tard, surtout si l'achat ne se "produit pas dès qu'il est possible et si l'ébullition est différée. "L'état bactériologique du lait, au moment de la pasteurisation et " au moment de l'ébullition chez le consommateur du même lait "resté cru est extrêmement différent.

" D'autre part, la pasteurisation véritable réalisée avant la "vente permet au consommateur d'emporter chez lui un lait " assaini et inoffensif. Les objets qui entreront en contact avec ce " lait (casseroles, cuillers, tétines...), de même que le sol ou les

(2) L'Industrie Laitière, n० 8 , août 1935. 
« tables qui pourront accidentellement recevoir quelques gouttes de "lait ne seront pas souillés d'une façon dangereuse.

"Au contraire avec un lait cru bacillifère, seules seront assainies "les portions de lait qui auront vraiment été soumises à l'action " d'une haute température. Les différents objets qui seront entrés en « contact avec le lait cru auront été souillés et seront capables de " contaminer le lait bouilli. Celui-ci redevient alors, dans une cer" taine mesure, un nouveau lait cru avec tous ses dangers éventuels.

"Avec un lait eru contaminé on peut affirmer qu'un danger est " entré dans la maison. Le danger du lait cru, dans le cas du con"sommateur qui le fait bouillir, est cette porte largement ouverte "aux souillures provenant de vaches ou de vachers malades. C'est " pourquoi il a pu être démontré, au cours de certaines épidémies " de fièvre typhoïde causées par le lait cru, que l'ébullition du lait " chez le consommateur n'a jamais empêché le microbe d'entrer dans " la maison et de contaminer secondairement le lait bouilli d'abord, "les personnes ensuite.

“ N'est-ce pas une opinion semblable qu'exprime le Professeur "Lereboullet quand il écrit : "Au surplus, les faits sont là qui " montrent les méfaits des laits trop pollués, alors même qu'ils ont "été soumis à l'ébullition."

“En somme, en dépit des apparences, il faut bien admettre que "l'ébullition chez le consommateur ne résout pas le problème de " l'hygiène du lait. Ce qu'il faut, e'est l'assainissement thermique le " plus précoce, le plus parfait avant tout transport et toute mise en "vente, par la pasteurisation."

\section{***}

La conclusion logique de ce qui vient d'être dit concernant la méthode curative, c'est-à-dire la pasteurisation, est la suivante : la pasteurisation apporte une solution certaine et efficace aux dangers d'ordre bactériologique présentés par le lait cru. Elle n'offre, par ailleurs, aucun inconvénient d'ordre diététique ou autre et sa valeur alimentaire est au moins égale à celle du lait cru.

Cette conclusion, basée sur des faits précis et des expériences sérieuses, est-elle partagée par les médecins, les hygiénistes, les autorités sanitaires ?

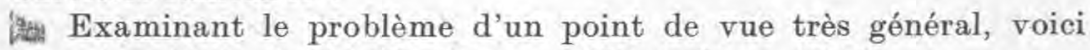
ce qu'en dit le Dr B. W. Hammer (Etats-Unis, 1948) :

"Comme résultat des épidémies rapportées au lait cru et de la " présence de germes pathogènes dans ce lait, les responsables du " contrôle de l'approvisionnement en lait commencent par réclamer " la pasteurisation de tout lait. Quand éclate une épidémie due au 
"lait cru, il y a une demande immédiate pour la pasteurisation du " lait destiné à la localité intéressée.

" De nombreuses personnes intéressées à la qualité hygiénique " des aliments insistent pour la pasteurisation du lait et cette "demande est appuyée, dans certaines circonstances, par des cam"pagnes éducatives mettant en évidence la valeur de la pasteurisa"tion.

"Le lait pasteurisé n'est plus eonfiné aux grandes villes. Il " constitue maintenant un pourcentage considérable de l'approvi"visionnement de nombreuses petites villes. Le public commence "généralement à apprécier le danger des maladies causées ou " répandues par le lait cru et à reconnaître la protection assurée par "la pasteurisation.

"Dans quelques circonstances des tentatives ont été faites, "pour satisfaire aux demandes de lait sain, de remplacer la pas"teurisation par le contrôle des vaches vis-à-vis de plusieurs mala"dies (tested herds). Mais les vaches malades ne sont qu'une des " sources d'entrée des germes pathogènes dans le lait. La pasteurisa"tion détruit les pathogènes présents dans le lait, quelle que soit "leur origine et, pour cette raison, est plus efficace que n'importe "quel système de contrôle du bétail. Les règlements qui requièrent " " soit la pasteurisation, soit le contrôle du bétail " ne tiennent pas " compte de la différence d'efficacité des deux procédés. "

Voici maintenant l'avis exprimé par le Professeur Maurice Lusr (Médecin Chef du Centre de Puériculture de Bruxelles) dans son "Traité de Diététique du Nourrisson " (1947):

"Dans certains pays, en Hollande par exemple, au début du "siècle, sous l'influence des hygiénistes, on créa de nombreuses " étables modèles qui produisaient un lait absolument irréprochable " et que l'on pouvait, quasi sans crainte, donner tel quel aux nourris"sons. De telles laiteries se sont également créées en France et en "Belgique ; elles ont toutes dû terminer leur activité par suite de " grandes difficultés financières. Le lait, pour qu'il puisse être donné " aux nourrissons sans avoir été stérilisé, pasteurisé ni même bouilli, " doit être produit et distribué avec des précautions techniques si " compliquées, que le prix de revient de ce lait est nettement trop "élevé, pour les bénéfices tout théoriques que le nourrisson peut en "tirer. "

"Les hygiénistes ont complètement modifié leur façon de voir à ce "sujet et insistent, actuellement, pour que l'Etat ou les Municipalités prennent les disposition, légales afin d'empêcher la vente du lait cru.

"Le premier souci des hygiénistes est de donner aux nourrissons " un aliment non nocif, c'est-à-dire exempt de germes pathogènes. 
" Pour l'obtenir, il est indispensable de porter le lait à une tempéra" ture telle que tous les germes nocifs soient.détruits.

"Il est un point sur lequel il est nécessaire d'insister, c'est "l'idée trop répandue dans les précis de vulgariasation du "Lait "vivant ». Etaient compris dans ce terme les diastases, ferments, " enzymes, oxydases et surtout les précieuses vitamines contenues "dans le lait cru (1). En donnant du "lait vivant" aux enfants, " on a souvent, hélas, donné du lait mortel... Comme le disait "Marfan, le lait cru de la vache est l'aliment normal du veau, " comme d'ailleurs le lait cru de la femme est celui de l'enfant. Si " celui-ci doit recourir au lait de vache, ce dernier doit être donné " cuit, c'est-à-dire modifié par la chaleur."

(Il semble, au reste, que la plupart des nourrissons digèrent " plus facilement le lait cuit que le lait cru (Nobecourt et Veslot).

"En somme, la consommation de lait cru ne présente pas (sauf " cas exceptionnel) d'avantage pour les nourrissons, surtout en " comparaison des risques que ce produit peut faire courir à l'en"fant. "

Les avis autorisés favorables à la pasteurisation du lait sont innombrables. Citons-en seulement quelques-uns que nous choisissons, à dessein, presque exclusivement aux Etats-Unis où, jadis, la consommation du lait cru était extrêmement répandue :

C. H. Eckles, W. B. Combs, J. Macy (Université de Minnesota, 1943) : "Partout où se pose la question de la qualité sanitaire d'un " approvisionnement en lait, la meilleure précaution à prendre est " soit d'écarter le lait suspect, soit de le pasteuriser convenablement. "La pasteurisation est l'une des plus valables sauvegardes que l'in"dustrie laitière puisse utiliser pour éviter la transmission des " maladies par le lait."

Dr McCollum, de l'Université Hopkins : "Toute ville devrait " rendre obligatoire la pasteurisation de sa. fourniture de lait. Les " motifs en sont clairs et sont admis par tous ceux qui peuvent com"prendre les principes de cette opération à la suite d'un enseigne" ment scientifique. Depuis des années, j'insiste pour que toutes les "villes fassent pasteuriser leur lait. "

Dr Thomas WooD, de l'Université de Colombia : « Actuellement, " me basant sur les faits que je connais, je suis d'avis que la pas" teurisation obligatoire de tout le lait est une mesure de protection " désirable pour l'hygiène publique. "

Dr L. R. Williams, de la National Tuberculosis Association de

(1) Nous avons montré plus haut comment les techniques modernes de pasteurisation préservent intégralement les vitamines naturelles du lait. 
New-York : "Nous recommandons constamment l'utilisation du " lait pasteurisé et dans nos publications nous insistons fréquemment "pour que le lait soit pasteurisé avant sa consommation."

$\mathrm{D}^{\mathrm{r}}$ Ch. Hastings, de Toronto (Canada) : "Il est inconcevable "qu'une personne pensante veuille, en présence de nos connaissances " actuelles, compromettre sa réputation en avançant des arguements "contre la pasteurisation scientifique du lait A mon avis, une "municipalité qui ne sauvegarde pas sa fourniture de lait au moyen «. de la pasteurisation scientifique est, à la lumière de la science "moderne, coupable de négligence criminelle."

Miss M. E. Murphy, du MeCormick Memorial Fund (Chicago) : "Il est évident que lorsque nos visiteuses conseillent le lait, elles "supposent qu'on donne aux enfants du lait pasteurisé. Nous vou"drions qu'il soit entendu une fois pour toutes, que nous jugeons « qu'il est dangereux de donner aux enfants du lait qui ne l'a pas "été. "

Dr A. JACOBI, Université de Columbia : " Le point le plus impor" tant pour les enfants en bas âge consiste simplement en ceci : "ne leur donnez pas de lait cru."

Dr Kelley, Commissaire du Département d'Hygiène de l'Etat de Massachusetts : "Le fait que le lait cru peut transmettre le " bacille virulent de la tuberculose et les organismes de la fièvre "typhoïde, de la fièvre scarlatine, de la diphtérie de l'angine sep"tique pour ne pas mentionner d'autres infections de moindre "importance, a décidé les autorités de l'Etat en général à recom" mander instamment et avec persistance une pasteurisation effi" cace. Le résultat fut que le pourcentage de lait pasteurisé de la " fourniture totale de lait a augmenté, en quatre ans, de 34 à $75 \%$ et " que nombre de grandes villes ont plus de $90 \%$ de lait pasteurisé. "Il en est résulté également que les épidémies des quatre affections " mentionnées sont devenues très rares."

A l'appui des déclarations précédentes, les résultats rassemblés par Feenster concernant le nombre des épidémies dues chaque année au lait dans l'Etat de Massachusetts, montrent leur régression continue, au fur et à mesure du développement de la pasteurisation : (moyennes annuelles)

$1911-1915 \ldots \ldots \ldots \ldots \ldots \ldots \ldots \ldots$

$1916-1920 \ldots \ldots \ldots \ldots \ldots \ldots \ldots$

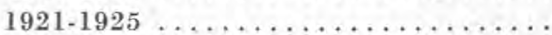

$1926-1930 \ldots \ldots \ldots \ldots \ldots \ldots \ldots$

1931-1935 ................

$1936-1940 \ldots \ldots \ldots \ldots \ldots \ldots \ldots \ldots$

$1941-1945$ 
Dr W. Peters, de Cincinnati : "Depuis décembre 1929 tout lait « certifié et inspecté doit être pasteurisé à Cincinnati à la suite d'un " ordre du Bureau d'Hygiène en coopération avec la Commission du " lait de l'Académie de Médecine et les producteurs. On a toujours " estimé les laits certifiés et inspectés comme de très bonne qualité. " Mais l'avis a prévalu qu'ils seraient bien meilleurs après la pas" teurisation, pasteurisation que nous considérons comme une " mesure de protection additionnelle."

Dr M. J. Rosenau, Professeur de Médecine préventive à l'Université de Harvard : "La pasteurisation sauve des vies et prévient " des maladies. Elle n'altère d'aucune façon la qualité du lait et ne " diminue pas sa valeur nutritive. La pasteurisation est la forme la " moins chère d'assurance sur la vie que le consommateur puisse "obtenir. "

Professeur R. M. WASHBURN (1931) : "Tous, nous sommes con" vaincus des avantages de la production hygiénique du lait, mais il " y a une lacune. Tout lait, même le lait le meilleur, même le lait "certifié, peut être infecté, devenir le véhicule d'une affection spéci" fique contagieuse et la communiquer à l'être humain. Ceci n'est pas "une supposition : ces faits se sont produits. "

Dr W. Huntington (parlant d'une épidémie de parathyphoïde à New-Rochelle) : "L'infection fut propagée par le lait d'une "laiterie productrice de lait certifié. Les affections contagieuses ne * sont pour ainsi dire jamais propagées par du lait qui est pasteurisé "dans les conditions requises."

Dr S. J. Crumbine, (American Child Health Association) : "Avec chacun des progrès nouveaux dans la connaissance des " maladies infectieuses, nous avons l'impression de nous trouver " devant une augmentation de danger pour la santé, en ce qui "concerne ces affections, lors de la consommation de lait cru."

Dr R. M. Ramsay, de Saint-Paul : "Je n'ai jamais constaté de " cas d'affection contagieuse qui puisse être attribué à du lait pas" teurisé dans des conditions appropriées. La baisse extraordinaire "de la mortalité infantile dans les grandes villes est directement "attribuable à la pasteurisation du lait et a démontré son utilité "d'une façon concluante."

Dr H. Bundesen, Commissaire d'Hygiène à Chicago : "Depuis "que la pasteurisation de tout lait (hormis le lait certifié) a été " rendue obligatoire en 1916 il n'y a pas eu un seul cas de contagion « qui puisse être attribué à la fourniture du lait : record qui vaut des "volumes sur l'efficacité de la pasteurisation comme mesure "publique. "

Voici, pour terminer, quelques avis de spécialistes français :

Professeur Lereboullet, Rapport au $3^{\text {e }}$ Congrès de Pédiatrie, 
Londres, 1933 : "Les inconvénients du lait cru, qui sont ses dangers " microbiens, ne sont pas niables. La pasteurisation du lait supprime "pratiquement les dangers de maladies qui pourraient résulter de "l'emploi du lait cru."

WARNERY, Institut Pasteur de Paris, 1934, parlant de la pasteurisation s'exprime ainsi : "Il s'agit là d'une méthode qui devrait "être généralisée et rendue obligatoire pour tout lait livré à la con"sommation."

Voici enfin, l'opinion de MARFAN (loc. cit., p. 566) : « Une obser" vation prolongée en ville et à l'hôpital nous permet de porter un "jugement favorable : avec le lait de bonne qualité, purifié par la "chaleur par une bonne méthode, les incidents de l'allaitement sont " très réduits, les augmentations de poids sont plus régulières, le " nombre des diarrhées diminue, surtout le nombre des diarrhées "graves... Ainsi l'emploi du lait chauffé a contribué à diminuer la " mortalité infantile... ")

De semblables citations d'avis concordants pourraient être multipliées à l'infini, émanant d'hygiénistes et de médecins du monde entier. Il semble, maintenant, superflu d'insister. L'unanimité s'est faite, surtout à l'étranger, sur les points suivants qui résultent de l'étude impartiale des faits :

1. Le lait cru constitue un danger par les germes de maladies qu'il peut renfermer ou propager.

2. Il est impossible, pratiquement, de porter remède à cette situation par une amélioration profonde et définitive concernant les animaux, les personnes, les opérations de récolte du lait.

3. La pasteurisation est d'une efficacité absolue vis-à-vis des inconvénients bactériologiques du lait cru.

4. Cette même opération (réalisée correctement, à l'abri de l'air, dans des appareils modernes) ne diminue en rien la digestibilité, la teneur vitaminique, la valeur alimentaire et diététique du lait. La croissance et le développement général des enfants alimentés au lait pasteurisé sont les mêmes que ceux des enfants nourris au lait cru. Parfois, même, ils sont meilleurs.

$$
* * *
$$

La cause est done entendue. Les spécialistes du monde entier ont apporté les preuves nécessaires. En maints endroits les autorités responsables recommandent la pasteurisation du lait ou la rendent obligatoire.

L'heure est venue de se demander où nous en sommes en France.

Certes, un mouvement s'est déclenché dans les milieux médi- 
caux et scientifiques français, depuis longtemps déjà, en faveur de la pasteurisation obligatoire du lait.

Ce mouvement a abouti à la loi du 2 juillet 1935 qui a posé le principe de cette obligation. Malheureusement cette loi a prévu des exemptions tellement importantes à l'application du principe, que celui-ci a perdu toute efficacité au point de vue de l'Hygiène Publique. En fait, de ce point de vue, la situation antérieure à 1935 est restée inchangée après la promulgation de la loi. Le problème n'a pas été résolu.

II appartient donc au législateur et aux autorités responsables de le reprendre aujourd'hui avec le souci de faire passer l'intérêt général, e'est-à-dire d'hygiène publique - avant les intérêts particuliers. La loi du 2 juillet 1935 est restée pratiquement inopérante du point de vue de la généralisation de la pasteurisation parce que certains parlementaires ont cru que cette mesure menaçait des intérêts particuliers. Cette manière de considérer le problème de la Santé Publique est néfaste. Elle résulte moins du souci louable de défendre des intérêts légitimes que de l'ignorance ou du parti pris. (N'a-t-on pas dit au cours de la discussion de la loi du 2 juillet 1935 , que " la pasteurisation n'était pas une méthode d'amélioration mais, tout simplement, un moyen de conservation "...)

Le législateur d'aujourd'hui, clairement informé, doit poser la question sur son véritable terrain, celui de l'Hygiène, et ne pas perdre de vue que "rendre la pasteurisation obligatoire, comme l'écrivait PoRcher, c'est satisfaire aux exigences de l'hygiène la mieux comprise ".

Il s'agit donc de sauvegarder ce principe en l'appliquant aussi largement que possible.

Sur le terrain pratique une objection apparaît immédiatement : de nombreuses fermes ne possèdent qu'un très petit nombre de vaches laitières, parfois une seule. Il paraît impossible de pasteuriser d'aussi petites quantités de lait ainsi produites.

Or, il convient d'insister sur le fait que plusieurs constructeurs français et étrangers ont mis au point des appareils de pasteurisation de très petit modèle permettant de traiter quelques dizaines de litres de lait. Cette fabrication, qui peut être largement développée, encouragée, aidée même, permettrait d'introduire la pasteurisation dans un très grand nombre de fermes qui vendent actuellement leur lait cru directement au consommateur et pourraient ainsi continuer leur exploitation en vendant leur lait pasteurisé.

Si le nombre de vaches est si faible que leṣ quantités de lait produites ne permettent pas l'emploi de ces appareils - même du plus petit modèle - la vente directe de ce lait cru au consommateur 
devrait alors être interdite. Il serait cédé à un ramasseur qui, lui, devrait obligatoirement pasteuriser (il pourrait aussi être cédé à une ferme plus importante soumise par définition à la même obligation). Nous rencontrons là la difficulté la plus sérieuse. On fera l'objection que les intérêts du petit producteur vont se trouver lésés par un prix de vente moins élevé. Il ne semble pas impossible cependant d'étudier une mesure compensatrice permettant de sauvegarder l'application du principe.

Il va de soi que, quelle que soit l'importance de la ferme, le producteur qui ne voudrait pas s'astreindre à la pasteurisation, devrait obligatoirement céder son lait cru à un ramasseur, à un groupement, à une société, etc., qui, tous, seraient tenus de procéder à la pasteurisation du lait.

Ne seraient finalement exemptés de la pasteurisation, que les laits produits dans des conditions hygiéniques spéciales (laits d'étables soumises au contrôle officiel vétérinaire et médical). Toutefois ne doivent pas être considérés comme laits répondant à cette condition, ceux qui résultent de mesures partielles (par exemple troupeaux tubereulinés) dont il a été nettement prouvé qu'ils n'étaient pas exemptś de dangers. Tout progrès partiel réalisé dans la production du lait est, certes, désirable et doit être encouragé. Mais il ne peut pas supprimer l'obligation de la pasteurisation. Il ne doit pas être perdu de vue en effet que la pasteurisation ne constitue pas une mesure dispendant de tout effort au niveau de la production. Ces efforts sont indispensables. Mais, sauf dans le cas particulier des laits d'étables contrôlées officiellement, ils sont nécessairement insuffisants et doivent être obligatoirement complétés par la pasteurisation.

On aboutirait ainsi à une véritable classification des laits qui serait la suivante :

a) Lait cru : provenant exclusivement d'étables soumises au contrôle officiel. Il s'agit done d'un lait cru certifié et contrôlé. II ne peut pas être envisagé d'autre qualité de lait cru pour la consommation humaine.

Encore convient-il de penser et de dire (d'accord en cela avec G. Thinulin, l'éminent hygiéniste dont les avis font autorité en la matière) que le lait cru ainsi défini, s'il peut être consommé librement par les adultes, ne devrait être donné aux jeunes enfants que sur ordonnance médicale.

b) Lait pasteurisé de grade A : résulte de la pasteurisation du lait cru ei-dessus défini (et vendu en bouteilles cachetées).

c) Lait pasteurisé de grade B : résulte de la pasteurisation du lait 
cru ordinaire non soumis au contrôle officiel mais également vendu en bouteilles cachetées.

C'est la qualité actuelle de ¿ lait pasteurisé certifié ".

d) Lait pasteurisé de grade C : résulte de la pasteurisation du lait eru ordinaire non soumis au contrôle officiel et vendu à la mesure. C'est la qualité actuelle de "lait pasteurisé ».

Cette manière d'envisager le problème du lait de consommation dans son ensemble paraîtra révolutionnaire à quelques-uns. Elle l'est, en effet, mais c'est dans le sens, très hautement désirable, de la sauvegarde de l'hygiène publique, de la diminution des maladies et de la mortalité infantiles. L'exemple donné par la ville de Strasbourg où la pasteurisation est obligatoire, est extrêmement significatif. Le rapporteur de la loi du 2 juillet 1935 disait à son sujet (séance de la Chambre des Députés du 28 mars 1935) :

"Je suis très heureux de rendre hommage à cette ville. A ( Strasbourg, en effet, la mortalité infantile était de $25 \%$ il y a quel"ques années. Depuis que les mesures d'hygiène que nous préconi« sons y ont été imposées, elle est tombée d'abord à $8 \%$. Elle est, " actuellement, de $5 \%$. C'est la ville où la mortalité infantile est la “ moins élevée après avoir été la plus forte. Il a suffi d'adopter des "mesures d'assainissement du lait pour qu'aussitôt la mortalité " infantile devienne quasi nulle. La ville de Strasbourg peut être, " à ce titre, citée à l'ordre du jour de la Nation."

Les députés qui ont applaudi ces paroles n'ont malheureusement pas compris que ce qui était bon et possible à Strasbourg, l'était aussi dans l'ensemble du pays. Ils se sont laissés détourner de leur devoir par des arguments fallacieux, erronés ou partisans présentés et défendus avec âpreté par certains députés plus soucieux d'intérêts électoraux que de l'hygiène de la Nation et de l'avenir de la race.

Combien d'enfants sont morts depuis seize ans parce que le Parlement de 1935 a failli à sa véritable mission?

Si le principe de la pasteurisation obligatoire comporte, certes, certaines difficultés d'application, la raison et l'intérêt général demandent que ces difficultés soient étudiées, résolues et non pas éludées par le maintien du déplorable état de choses actuel.

La France, pays de PASTÉuR, se doit de passer au premier plan dans le domaine de l'hygiène du lait de consommation : la pasteurisation obligatoire en est le moyen. 